\title{
Experimental investigation of Reynolds stress complex eddy viscosity model for coherent structure dynamics
}

\author{
JIA YongXia ${ }^{2}$, TANG ZhanQi ${ }^{2} \&$ JIANG Nan ${ }^{1,2,3 *}$ \\ ${ }^{1}$ State Key Laboratory of Nonlinear Mechanics, Institute of Mechanics, Chinese Academy of Sciences, Beijing 100080, China; \\ ${ }^{2}$ Department of Mechanics, School of Mechanical Engineering, Tianjin University, Tianjin 300072, China; \\ ${ }^{3}$ Tianjin Key Laboratory of Modern Engineering Mechanics, Tianjin 300072, China
}

Received October 21, 2010; accepted February 21, 2011; published online May 30, 2011

\begin{abstract}
Time sequence signals of streamwise and normal velocity components, as well as velocity strain rate, at different vertical locations in the turbulent boundary layer over a smooth flat plate in a wind tunnel have been finely examined by the use of double-sensor hot-wire anemometry. The local module maximum for wavelet coefficient of longitudinal velocity component, as a detecting index, is employed to educe the ejection and sweep process of the coherent structure burst in the turbulent boundary layer from the random fluctuating background. The coherent waveforms of Reynolds stress residual contribution term for random fluctuations to coherent structure, as well as the velocity strain rate of coherent structure, are extracted by the conditional phase average technique. Based on the theoretical analysis of eddy viscosity coefficient in complex eddy viscosity model for coherent structure, the macro-relaxation effect between Reynolds stress residual contribution term of random fluctuations to coherent structure and the velocity strain rate of coherent structure is studied and the variations of the phase difference between them across the turbulent boundary layer are investigated experimentally. The rationality of complex eddy viscosity model for coherent structure is confirmed through the investigation.
\end{abstract}

turbulent boundary layer, coherent structure, complex eddy viscosity model, Reynolds stress, phase difference

PACS: 47.27.De, 47.27.em, 47.27.nb, 47.80.Cb, 47.11.St

\section{Introduction}

In the earlier stage of turbulence studies, turbulence was considered as completely random motion of fluid particles. The theory of turbulence model, in order to avoid difficulties in the study of randomness, was established for solving the turbulent ensemble average movement quantity as a primary purpose. However, with the starting of Reynolds' (1895) decomposition of the turbulent flow variables into mean and fluctuating two parts, the approach of the Reynolds-averaged Navier-Stokes (RANS) equations gives rise to the unknown Reynolds stress tensor $-\rho \overline{u_{i}^{\prime} u_{j}^{\prime}}$, and leads to

*Corresponding author (email: nanj@tju.edu.cn) the closure problem of RANS equations. In order to enclose RANS equations, the Reynolds stress model should be introduced relying on a combination of theory and experience by employing a series of quantities related to the mean flow field. So the important role of Reynolds stress in the turbulent model has stimulated attempts to reveal the physical mechanisms of turbulence in the production and transport process. The results of DNS and experiments show that the fundamental mechanisms of Reynolds stress production are closely related to the dynamics of coherent structure (CS) in the near wall region. An in-depth research study on the Reynolds stress evolution during CS burst should be spurred for establishing realistic Reynolds stress models. However, current descriptions for the physical process, especially the dynamics aspects, remain preliminary. 
In the turbulent flow field, there are both multi-scale CS and random fluctuation components besides the mean flow. Any physical quantity describing a turbulent flow field can be divided into three parts [1]:

$$
f=\bar{f}+\tilde{f}+f^{\prime},
$$

where $\bar{f}$ stands for the mean part, $\tilde{f}$ represents the coherent part and $f^{\prime}$ corresponds to the random fluctuation with $\tilde{f}$ and $f^{\prime}$ being statistically independent. Then, the phase average component is

$$
\langle f\rangle=\bar{f}+\tilde{f} .
$$

For incompressible constant-property flow, the continuity and momentum Navier-Stokes equations are written as:

$$
\frac{\partial u_{i}}{\partial x_{i}}=0
$$

and

$$
\frac{\partial u_{i}}{\partial t}+u_{j} \frac{\partial u_{i}}{\partial x_{j}}=-\frac{1}{\rho} \frac{\partial p}{\partial x_{i}}+v \frac{\partial}{\partial x_{j}} \frac{\partial}{\partial x_{j}} u_{i}
$$

The velocity and pressure fields can be decomposed as:

$$
\begin{gathered}
u_{i}=\bar{u}_{i}+\tilde{u}_{i}+u_{i}^{\prime}, \\
p=\bar{p}+\tilde{p}+p^{\prime} .
\end{gathered}
$$

After substituting eqs. (5) and (6) into eq. (4) and ensemble averaging, the equations for the mean flow are given as:

$$
\begin{aligned}
& \frac{\partial \overline{u_{i}}}{\partial t}+\overline{u_{j}} \frac{\partial \overline{u_{i}}}{\partial x_{j}}=-\frac{1}{\rho} \frac{\partial \bar{p}}{\partial x_{i}} \\
& +v \frac{\partial}{\partial x_{j}} \frac{\partial}{\partial x_{j}} \overline{u_{i}}-\frac{\partial}{\partial x_{j}}\left(\overline{u_{i}^{\prime} u_{j}^{\prime}}+\overline{\tilde{u}_{i} \tilde{u}_{j}}\right) .
\end{aligned}
$$

After substituting eqs. (5) and (6) into eq. (4) and phase averaging, then subtracting eq. (7), the dynamical equations for CS are expressed as:

$$
\begin{aligned}
\frac{\partial \tilde{u}_{i}}{\partial t}+\bar{u}_{j} \frac{\partial \tilde{u}_{i}}{\partial x_{j}}+\tilde{u}_{j} \frac{\partial \bar{u}_{i}}{\partial x_{j}} & -\frac{1}{\rho} \frac{\partial \tilde{p}}{\partial x_{i}}+v \frac{\partial}{\partial x_{j}} \frac{\partial}{\partial x_{j}} \tilde{u}_{i} \\
& -\frac{\partial}{\partial x_{j}}\left(\left\langle u_{i}^{\prime} u_{j}^{\prime}\right\rangle-\overline{u_{i}^{\prime} u_{j}^{\prime}}\right)-\frac{\partial}{\partial x_{j}}\left(\left\langle\tilde{u}_{i} \tilde{u}_{j}\right\rangle-\overline{\tilde{u}_{i} \tilde{u}_{j}}\right) .
\end{aligned}
$$

Eq. (8) describes CS motion. However, in eq. (8) there appears a term:

$$
\tilde{r}_{i j}=-\left(\left\langle u_{i}^{\prime} u_{j}^{\prime}\right\rangle-\overline{u_{i}^{\prime} u_{j}^{\prime}}\right),
$$

which is unknown. $\tilde{r}_{i j}$ represents the Reynolds stress residual contribution from random fluctuation to CS except to the mean flow. In this paper $\tilde{r}_{i j}$ will be modeled by complex eddy viscosity model (CEVM) (eq. (10)):

$$
\tilde{r}_{i j}=2 v_{T} \tilde{S}_{i j}+\frac{1}{3} \delta_{i j}\left(\tilde{r}_{k k}\right),
$$

where $\tilde{S}_{i j}=\frac{1}{2}\left(\frac{\partial \tilde{u}_{i}}{\partial x_{j}}+\frac{\partial \tilde{u}_{j}}{\partial x_{i}}\right)$. Eq. (10) in the two-dimensional form is expressed as:

$$
\begin{gathered}
\tilde{r}_{11}=v_{T}\left[\frac{\partial \tilde{u}}{\partial x}+\frac{\partial \tilde{u}}{\partial x}\right]+\frac{1}{3}\left(\tilde{r}_{11}+\tilde{r}_{22}+\tilde{r}_{33}\right), \\
\tilde{r}_{12}=v_{T}\left[\frac{\partial \tilde{u}}{\partial y}+\frac{\partial \tilde{v}}{\partial x}\right], \\
\tilde{r}_{22}=v_{T}\left[\frac{\partial \tilde{v}}{\partial y}+\frac{\partial \tilde{v}}{\partial y}\right]+\frac{1}{3}\left(\tilde{r}_{11}+\tilde{r}_{22}+\tilde{r}_{33}\right),
\end{gathered}
$$

where $v_{T}$ is the complex eddy viscosity coefficient, $u$ and $v$ are streamwise and vertical velocity components, respectively.

For the enclosure of Reynolds stress residual contribution term from random fluctuation to CS, $\tilde{r}_{i j}$, Reynolds et al. [1] proposed the quasi-laminar model $\left(\tilde{r}_{i j}=0\right)$, which means the influence of background turbulence stress on CS could be neglected, and does not exactly describe the behavior of CS in wall turbulence. They further advanced the Newtonian eddy model, and the use of a scalar eddy viscosity simulating $\tilde{r}_{i j}$ as $v_{T} \tilde{S}_{i j}$ which gave results different from those obtained with the 'actual' variable eddy viscosity. Wu et al. [2] adopted the constant CEVM, but the eigenfunction showed an oscillatory behavior, which was inconsistent with Brown's experimental results [3]. Falco et al. [4] presented a structural model of 'typical eddy' formed on the upstream of large scale motions, and found there was a phase relationship between 'typical eddies' producing most of the Reynolds stress and large scale motions associated with significant Reynolds stress contributions. Luo et al. [5] suggested a CEVM model based on the numerical simulation to explain large scale CS in the outer region of turbulent boundary layer (TBL). This CEVM model introduced a complex eddy viscosity coefficient as a function of normal-wall location and led to the numerical results consistent with experimental results of Antonia [6].

CEVM indicates a phase relationship between $\tilde{r}_{i j}$ and $\tilde{S}_{i j}$ due to the relaxation effect of turbulent eddy motions. As we know, the spatio-temporal scales of turbulent eddy motions during the turbulent momentum exchange process, compared with those of the molecular motions, need to be 
taken into account as turbulent eddy deformations never synchronize with Reynolds stress production both in time and space. The spatio-temporal relaxation effect between the eddy strain rate and the Reynolds stress in turbulent flows should not be neglected. This phenomenon is more remarkable in many periodic turbulent flows such as turbine flows. Hadzic et al. [7] found that the phase lag between the Reynolds stress and the strain rate tensors was particularly essential in the case when the flow was subjected to periodic strain of relatively high frequency and the lack of this feature led to continuous over-generation of turbulent kinetic energy. The phase information of turbulent fields therefore should be included in the eddy viscosity model. This proposal has been confirmed by the numerical simulations of $\mathrm{Wu}[2]$, Luo [5,8] and experimental measurements of Falco [4], Bruns [9], Wang [10], Li [11] and Guo [12]. However, the theoretical model of CS still needs to be further investigated to gain more detailed information in the different regions of the whole TBL.

A CEVM for simulating Reynolds stress was proposed by Luo [8] and Wang [10]. The eddy viscosity coefficient is denoted by

$$
v_{T}=\left|v_{T}\right| \mathrm{e}^{\mathrm{i}[\varphi(y)-\theta(y)]},
$$

$\varphi(y)$ is the phase function of Reynolds stress, $\theta(y)$ is the phase function of mean strain rate as the normal-wall location $y$ respectively.

In this paper, based on the CEVM formula $\tilde{r}_{12}=v_{T}[\partial \tilde{u} / \partial y+\partial \tilde{v} / \partial x]$ (where $\partial \tilde{v} / \partial x$ is negligible compared with $\partial \tilde{u} / \partial y)$, the measured phase information between $\tilde{r}_{12}$ and $\partial \tilde{u} / \partial y$ across the TBL was investigated by the hot-wire experimental technology, and the rationality of CEVM is further verified.

\section{Experimental apparatus and technique}

The experiments were performed in a low-speed close-circuit wind tunnel of Tianjin University. The test section is $1.5 \mathrm{~m}$ in length, $0.6 \mathrm{~m}$ in height and $0.8 \mathrm{~m}$ in width. A TBL flow had been established along a horizontally mounted stainless-steel plate $1.2 \mathrm{~m}$ long, $0.8 \mathrm{~m}$ wide and $2 \mathrm{~mm}$ thick, with a wedged leading edge, while a zero pressure gradient had been adapted by a trailing edge flap. The free-stream velocity was $U_{\infty}=8.9 \mathrm{~m} / \mathrm{s}$. The TBL was tripped by a sand-paper on the leading edge with a nominal thickness of $0.084 \mathrm{~m}$ corresponding to $\operatorname{Re}_{\delta}=\rho \mathrm{U}_{\infty} \delta / \mu=47467$ at the measurement location $800 \mathrm{~mm}$ downstream of the leading edge. The co-ordinate system is as follows: the $x$ axis is parallel to the free stream, the $y$ axis is perpendicular to the plate and the $z$ axis is perpendicular to both the $x$ and $y$ axes according to the conventional right-hand rule, correspond- ing to velocity components $u, v$, and $w$, respectively.

The IFA300 constant-temperature hot-wire anemometer with a double-sensor probe was used to carry out velocity measurements in the TBL. The sensor is tungsten wires with $5 \mu \mathrm{m}$ in diameter. A TSI-1243-T1.5 X-type probe was used to measure $u$ and $v$ components. Herein, the hot wires were inclined by $\pm 45^{\circ}$ towards the streamwise direction. A TSI-1244-T1.5 double-parallel-wire probe used to measure $\partial u / \partial y$ was mounted above the plate, facing the free stream. Each wire of the probe was calibrated independently to obtain the frequency response and the relationship between the output voltage and the flow velocity before measurement. The anemometer outputs were digitized at a sampling rate $f_{\text {acq }}=50 \mathrm{kHz}$ by an $\mathrm{A} / \mathrm{D}$ converter, and the raw data was stored online in a computer for post processing. To evaluate the mean flow field of the TBL, we used a TSI-1211-T1.5 single-wire probe to measure the longitudinal mean velocity profile. The semi logarithmic mean velocity profile normalized by viscous wall unit is given in Figure 1, where $u^{+}=u / u_{*} y^{+}=y u_{*} / v$. It can be seen from Figure 1 that the turbulent flow field is a standard TBL. The skin friction velocity estimated by regression between $y^{+}=40$ and $y^{+}=200$ is $u_{\tau}=0.3906 \mathrm{~m} / \mathrm{s}$ and the skin friction coefficient is $c_{f}=$ 0.0039 . Buffer layer, log-layer and bulk region can be distinguished in the single wall distance regions by their characteristic curvatures, while the linear viscous sub-layer region could not be resolved sufficiently. A TSI-1243-T1.5 $\mathrm{X}$-type probe was applied for instantaneous velocity measurements at a sampling rate of $50 \mathrm{kHz}$ at 102 locations across the TBL. Then, the corresponding velocity strain rate was also gained by a TSI-1244-T1.5 parallel-wire probe at all the same positions with the same experimental parameters. Figure 2 shows the streamwise and vertical turbulent intensity profiles across the TBL. Figure 3 presents the

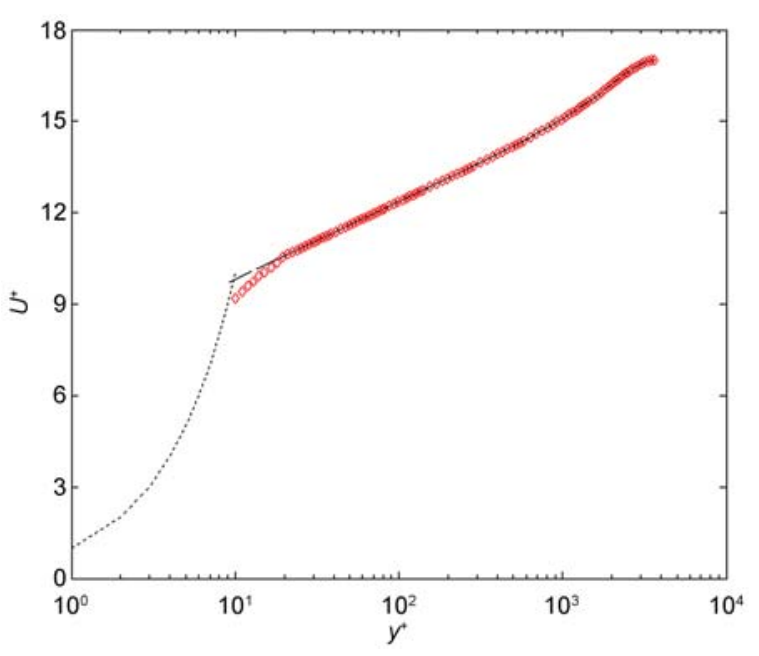

Figure 1 (Color online) Mean velocity profile of the TBL in wall viscous units: ------, $U^{+}=y^{+} ;-$, Coles law; $\diamond$, Exp. Data. 


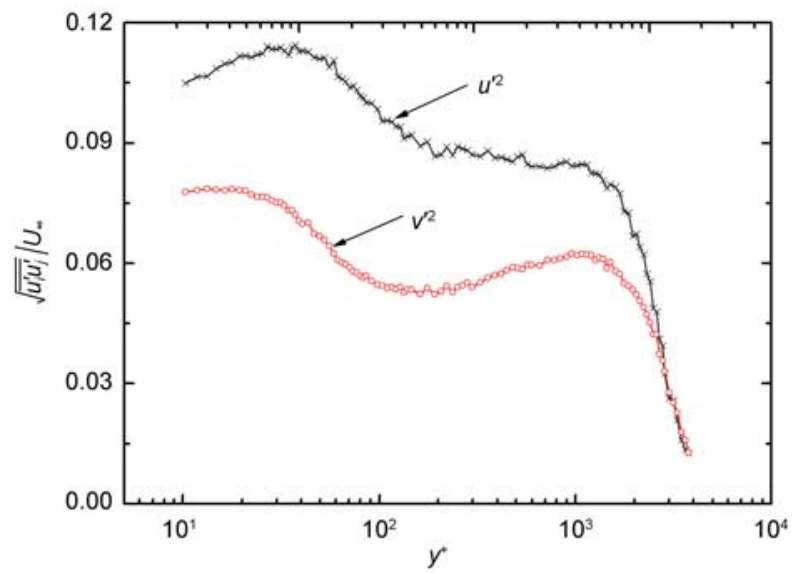

Figure 2 (Color online) Streamwise and vertical turbulent intensity profiles across the TBL. $u^{\prime}, v^{\prime}$ are the streamwise and vertical velocity fluctuations.

mean streamwise velocity strain rate profile across the TBL.

\section{Experimental results analysis}

In the present work, turbulent velocity signals will be decomposed into multiple-temporal-scale components by wavelet transform [13]. Wavelet transform (WT) is an effective mathematical technique widely used in the analysis of turbulent signals $[14,15]$, and allows us to analyze a turbulent flow in terms of space and scale. It is generally recognized that a turbulent field is a set of CS (such as hairpin vortices). In this case it will be more appropriate to decompose the flow field into different temporal scale CS by WT. WT of a turbulent instantaneous velocity field describes locally averaged deformation of CS with definite scale and limited scope at a certain location. Now we can take an example of Harr-WT for depicting the motion process of CS.

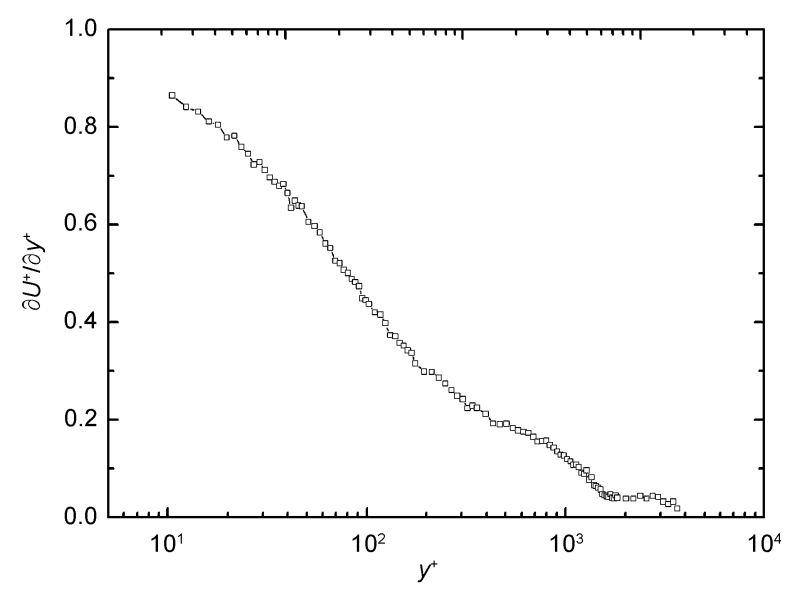

Figure 3 Mean streamwise velocity gradient profile in wall viscous units across the TBL.
WT of one-dimension turbulent velocity signal $u(x)$ is

$$
W_{\mathrm{H}}\left(a_{j}, b\right)=\frac{1}{\sqrt{a_{j}}}\left[\int_{b}^{b+a_{j}} u(x) \mathrm{d} x-\int_{b-a_{j}}^{b} u(x) \mathrm{d} x\right],
$$

where $j$ is the level of WT, $a_{j}$ is the stretching or compressing scale. $W_{\mathrm{H}}\left(a_{j}, b\right)$ may be interpreted as the relative difference between locally averaged velocities in the range of $x \in\left[b, b+a_{j}\right]$ and $x \in\left[b-a_{j}, b\right]$. For CS of space scale $2 a_{j}$, the case $W_{\mathrm{H}}\left(a_{j}, b\right)>0$ represents the stretching process of CS due to the upstream averaged convective velocity being smaller than downstream while the case $W_{\mathrm{H}}\left(a_{j}, b\right)<0$ exhibits the compressing process of CS owing to the upstream averaged convective velocity being larger than the downstream. The physical meaning of Harr-WT of turbulent velocity signals is consistent with the concept of locally averaged velocity structure function [16]:

$$
\delta u\left(a_{j}, b\right)=\overline{u(x)}_{x \in\left[b, b+a_{j}\right]}-\overline{u(x)}_{x \in\left[b-a_{j}, b\right]} .
$$

Figure 4 presents the time trace of instantaneous streamwise velocity and the contour of corresponding wavelet coefficients at $y^{+}=29.95$. From the standard $(j, t)$ plane representation of the wavelet coefficients, it can be seen that there exists one-to-one CS burst at different locations and scales compared with the velocity signal $u(t)$. The alternation of the red spots followed by the blue spots in the contour of wavelet coefficients is related to CS burst which may be the most important structures in wall turbulence and contribute most to the turbulence production in the near wall region. The red spot represents the accelerating events of different scales CS in which the high-speed fluids sweep the probe causing the high velocity output from the hot-wire probe while the blue spots stand for the decelerating events in which the low-speed fluids eject from the near wall region to the probe causing the low velocity output from the hot-wire probe. Figure 5 shows the time trace signal of instantaneous vertical velocity and the contour of corresponding wavelet coefficients at $y^{+}=29.95$.

Mean turbulent fluctuation kinetic energy of different scales CS obtained through WT is defined by

$$
\begin{aligned}
E\left(a_{j}\right) & =\overline{\left|W_{\mathrm{H}}\left(a_{j}, b\right)\right|^{2}} \\
& =\overline{\left|\frac{1}{\sqrt{a_{j}}}\left[\int_{-a_{j}+b}^{b} u(t) \mathrm{d} t-\int_{b}^{a_{j}+b} u(t) \mathrm{d} t\right]\right|^{2}} .
\end{aligned}
$$

Figure 6 plots the mean energy intensity distribution $E\left(a_{j}\right)$ vs. scale $j$ in the near wall region. The energy intensity distribution varies with scale $j$, and has a peak at the scale $j=8$, which indicates that the large-scale CS appears in the near wall region of TBL. Especially, the buffer layer shows significance in the TBL where the energy intensity is the most distinguishing. The result is attributed as CS being fairly active in this region and playing a quite important role of 

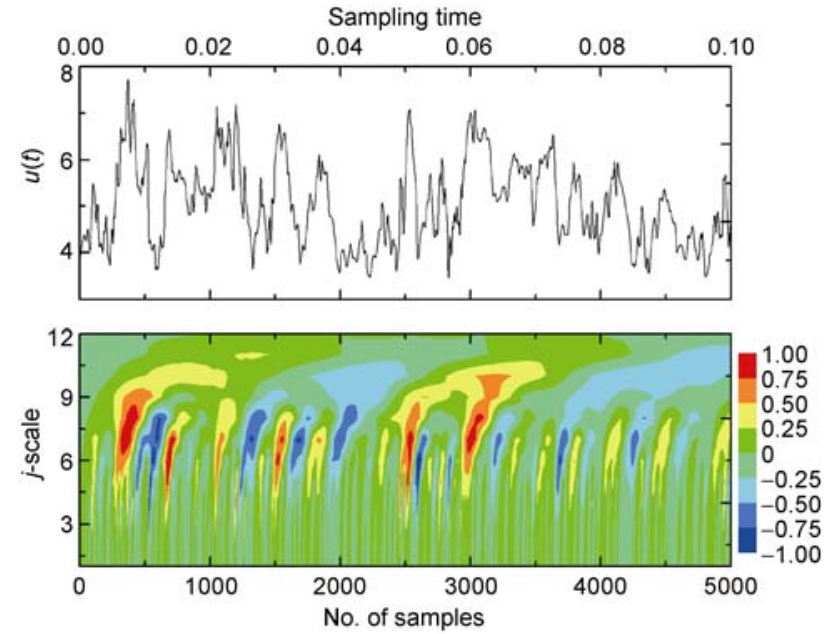

Figure 4 Streamwise velocity $u(t)$ (top) and contour of corresponding wavelet coefficients (bottom) at $y^{+}=29.95$.
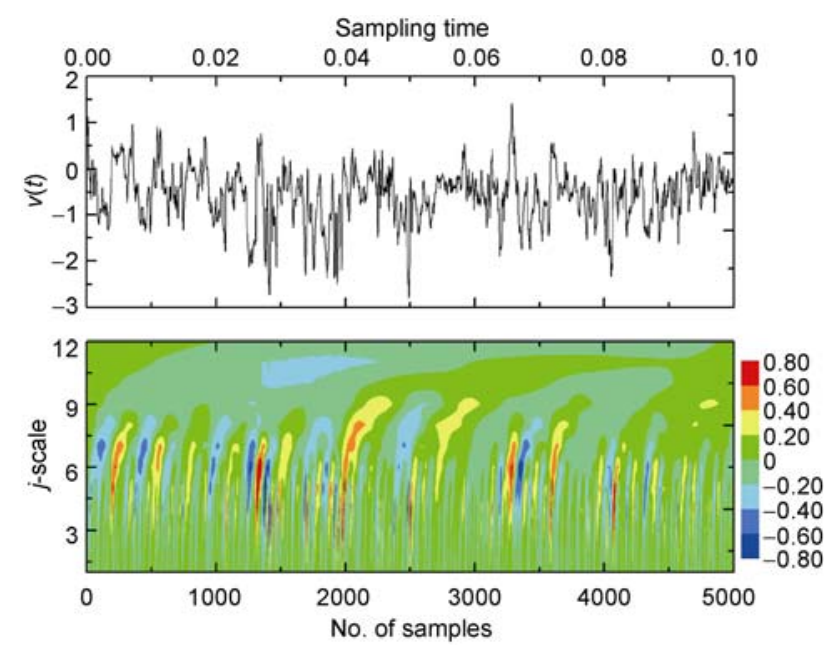

Figure 5 Vertical velocity $v(t)$ (top) and contour of corresponding wavelet coefficients (bottom) at $y^{+}=29.95$.

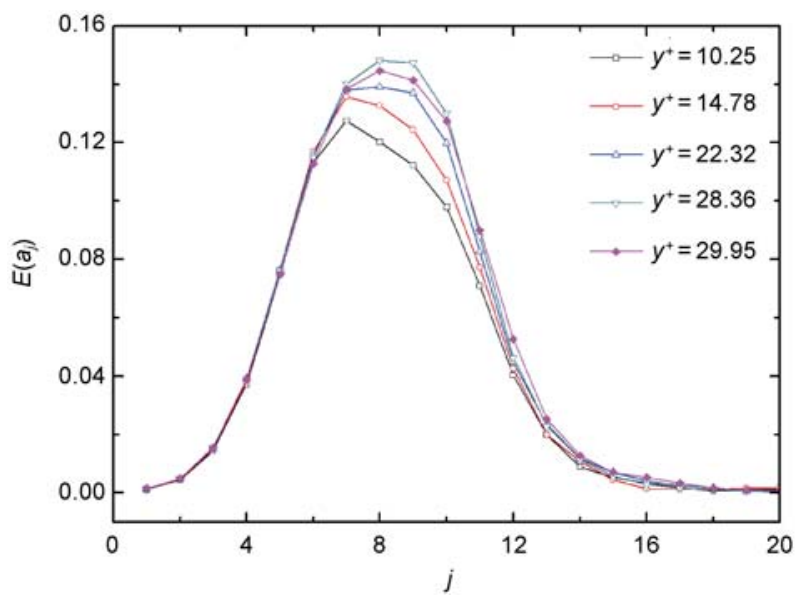

Figure 6 Energy distribution $E\left(a_{j}\right)$ vs. scale $j$ at different vertical locations of TBL. turbulence production.

To obtain the duration $T_{j}$ for $j$-th scale CS, we calculate the time autocorrelation of $j$-th scale wavelet coefficient. Figure 7 shows autocorrelation coefficients $\operatorname{cov}(t)$ vs. $t$ curves for $j$-th scale CS. $T_{j}$ corresponds to the wavelength between the two troughs of autocorrelation coefficient wave.

The $j$-th scale CS in the TBL is detected by the instantaneous modulus maximum of the wavelet coefficient:

$$
\begin{aligned}
& D_{\mathrm{e}}\left(a_{j}, t_{i}+t\right) \\
& =\left\{\begin{array}{l}
1, \quad t \in\left[-\frac{T_{j}}{2}, \frac{T_{j}}{2}\right], \\
\text { while } W\left(a_{j}, t_{i}\right)<0 \& W\left(a_{j}, t_{i}\right)=\text { min, } \\
0, \quad \text { otherwise, }
\end{array}\right. \\
& D_{\mathrm{s}}\left(a_{j}, t_{i}+t\right) \\
& = \begin{cases}1, & t \in\left[-\frac{T_{j}}{2}, \frac{T_{j}}{2}\right], \\
\text { while } W\left(a_{j}, t_{i}\right)>0 \& W\left(a_{j}, t_{i}\right)=\max , \\
0, & \text { otherwise, }\end{cases}
\end{aligned}
$$

where subscripts e and s represent ejection and sweep, respectively, and $D$ represents detected function. Then, the phase average components $\left\langle f\left(a_{j}, t\right)\right\rangle$ (f may represent $\left.u, v\right)$ of $j$-th scale CS in eject and sweep events are extracted through the conditional phase average (CPA) technique, to obtain CPA waveforms of $f$ :

$$
\begin{array}{r}
\left\langle f\left(a_{j}, t\right)\right\rangle_{\mathrm{e}}=\frac{1}{N_{j}} \sum_{i=1}^{N_{j}} f\left(t_{i}+t\right), t \in\left[-\frac{T_{j}}{2}, \frac{T_{j}}{2}\right], \\
\text { while } D_{\mathrm{e}}\left(a_{j}, t_{j}+t\right)=1, \\
\left\langle f\left(a_{j}, t\right)\right\rangle_{\mathrm{s}}=\frac{1}{N_{j}} \sum_{i=1}^{N_{j}} f\left(t_{i}+t\right), t \in\left[-\frac{T_{j}}{2}, \frac{T_{j}}{2}\right], \\
\text { while } \quad D_{\mathrm{s}}\left(a_{j}, t_{i}+t\right)=1,
\end{array}
$$

where $N_{j}$ is the number of $j$-th scale CS ejection or sweep events, and $T_{j}$ represents the duration of $j$-th scale CS shown in Figure 7. Then, the random fluctuation part $f^{\prime}$ is obtained by subtracting eq. (17) from eq. (1):

$$
f^{\prime}=f-\langle f\rangle=f-\bar{f}-\tilde{f} .
$$

The CPA waveforms of $\left\langle u^{\prime} v^{\prime}\right\rangle_{\mathrm{e}}$ and $\left\langle u^{\prime} v^{\prime}\right\rangle_{\mathrm{s}}$ are then extracted by the CPA technique:

$$
\begin{gathered}
\left\langle u^{\prime}\left(a_{j}, t\right) v^{\prime}\left(a_{j}, t\right)\right\rangle_{\mathrm{e}}=\frac{1}{N_{j}} \sum_{i=1}^{N_{j}} u^{\prime}\left(t_{i}+t\right) v^{\prime}\left(t_{i}+t\right), \\
t \in\left[-\frac{T_{j}}{2}, \frac{T_{j}}{2}\right], \text { while } D_{\mathrm{e}}\left(a_{j}, t_{j}+t\right)=1,
\end{gathered}
$$




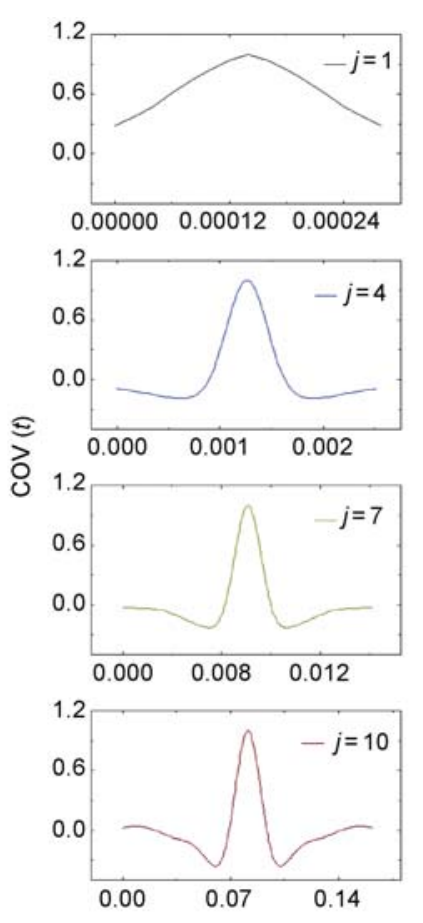

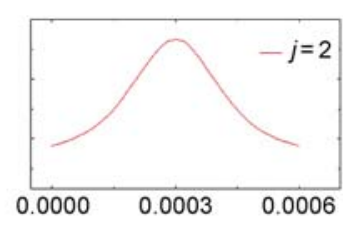
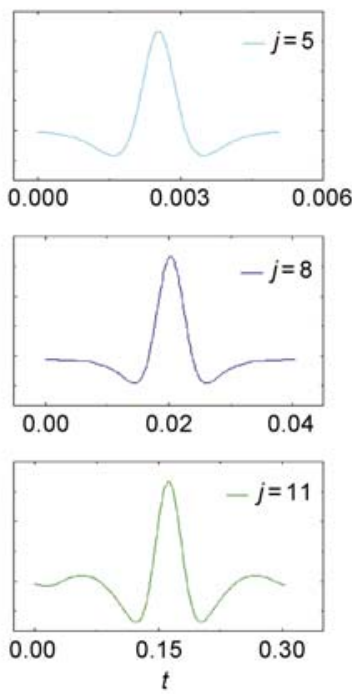
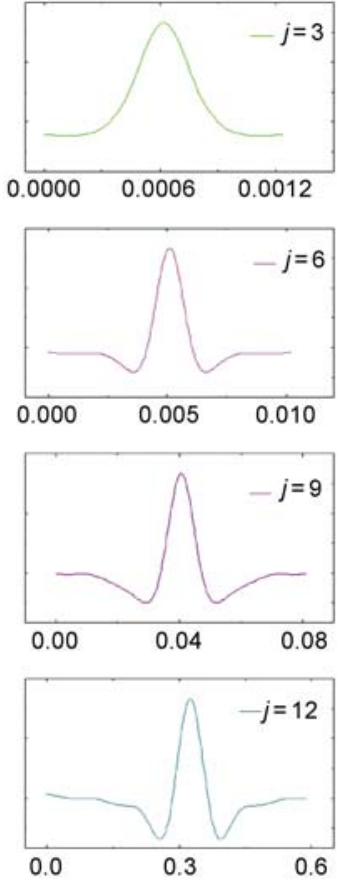

Figure 7 (Color online) Auto-correlation waves of the wavelet coefficients for $j$-th scale CS duration of $j$-th scale CS.

$$
\begin{gathered}
\left\langle u^{\prime}\left(a_{j}, t\right) v^{\prime}\left(a_{j}, t\right)\right\rangle_{\mathrm{s}}=\frac{1}{N_{j}} \sum_{i=1}^{N_{j}} u^{\prime}\left(t_{i}+t\right) v^{\prime}\left(t_{i}+t\right), \\
t \in\left[-\frac{T_{j}}{2}, \frac{T_{j}}{2}\right], \text { while } D_{\mathrm{s}}\left(a_{j}, t_{j}+t\right)=1,
\end{gathered}
$$

$\overline{u^{\prime} v^{\prime}}$ can be also obtained by the ensemble average of $u^{\prime} v^{\prime}=(u-\langle u\rangle)(v-\langle v\rangle)$. Then, $\tilde{r}_{12}=-\left(\left\langle u^{\prime} v^{\prime}\right\rangle-\overline{u^{\prime} v^{\prime}}\right)$ can be obtained by the difference between $\left\langle u^{\prime} v^{\prime}\right\rangle$ and $\overline{u^{\prime} v^{\prime}}$. In addition, the difference scheme is applied to calculate $\partial \tilde{u} / \partial y$ :

$$
\begin{aligned}
\frac{\partial \tilde{u}}{\partial y} & \approx \frac{\tilde{u}_{n+1}-\tilde{u}_{n}}{y_{n+1}-y_{n}} \\
& =\frac{(\langle u\rangle-\bar{u})_{n+1}-(\langle u\rangle-\bar{u})_{n}}{y_{n+1}-y_{n}} .
\end{aligned}
$$

In the current work the most energetic scale $(j=8)$, adequately representing the character of $\mathrm{CS}$, is chosen for presenting the CPA results. The CPA waveforms of $\tilde{r}_{12}$ and $\partial \tilde{u} / \partial y$ for 8-th scale CS during the ejection and sweep process in the logarithm layer are given in Figure 8(a)-8(d). One can find that there exists a phase difference between $\tilde{r}_{12}$ and $\partial \tilde{u} / \partial y$, viz. $\Delta \Phi=\varphi(y)-\theta(y)$, where $\varphi(y)=$ $2 \pi t / T_{j}$ and $\theta(y)=2 \pi t / T_{j}$ are the phase of $\tilde{r}_{12}$ and $\partial \tilde{u} / \partial y$ as functions of $y^{+}$, respectively. CPA waveforms of $\tilde{r}_{12}$ lag behind $\partial \tilde{u} / \partial y$ in phase, namely $\Delta \Phi<0$, during an ejection process. On the contrary, the phase of $\tilde{r}_{12}$ is ahead of $\partial \tilde{u} / \partial y$, namely $\Delta \Phi>0$, during a sweep process. The reason is that $\partial \tilde{u} / \partial y$ was mainly contributed by the spanwise vortex head of horseshoes CS which leads to the significant variation of $\tilde{u}$ in the normal-wall direction by its clockwise-spanwise rotation. Large-scale CS can depress local small-scale fluctuations. At the place where large-scale CS inhabits, local small-scale fluctuations (i.e. $\tilde{r}_{12}$ ) will be weakened. $\tilde{r}_{12}$ can only inhabit upstream and downstream of large-scale CS. Consequently, there exists the spatial phase difference between $\tilde{r}_{12}$ for small-scale fluctuation and $\partial \tilde{u} / \partial y$ for large-scale CS.

$\tilde{r}_{12}$ is mainly contributed by small-scale fluctuations of low-speed fluids ejected between the CS two vortex legs. The low-speed fluids rise up from the near wall low-speed streak by the positive normal-wall fluctuation velocity component $v^{\prime}>0$. So in the spatial streamwise direction, they will convect more slowly than CS with the relatively negative streamwise fluctuation velocity component $u^{\prime}<0$. They are relatively towards upstream of CS and lead $\tilde{r}_{12}$ to locate upstream of $\partial \tilde{u} / \partial y$. In the temporal direction, the phase of $\partial \tilde{u} / \partial y$ is ahead of that of $\tilde{r}_{12}$ and $\partial \tilde{u} / \partial y$ can be detected by the hot-wire probe earlier than $\tilde{r}_{12}$ so that $\theta(y)>\varphi(y)$ and $\Delta \Phi=\varphi(y)-\theta(y)<0$.

On the other hand, during sweep, clockwise rotation of spanwise hairpin vortex head of CS induces more high-speed small-scale fluctuations to $\tilde{r}_{12}$. They will con- 

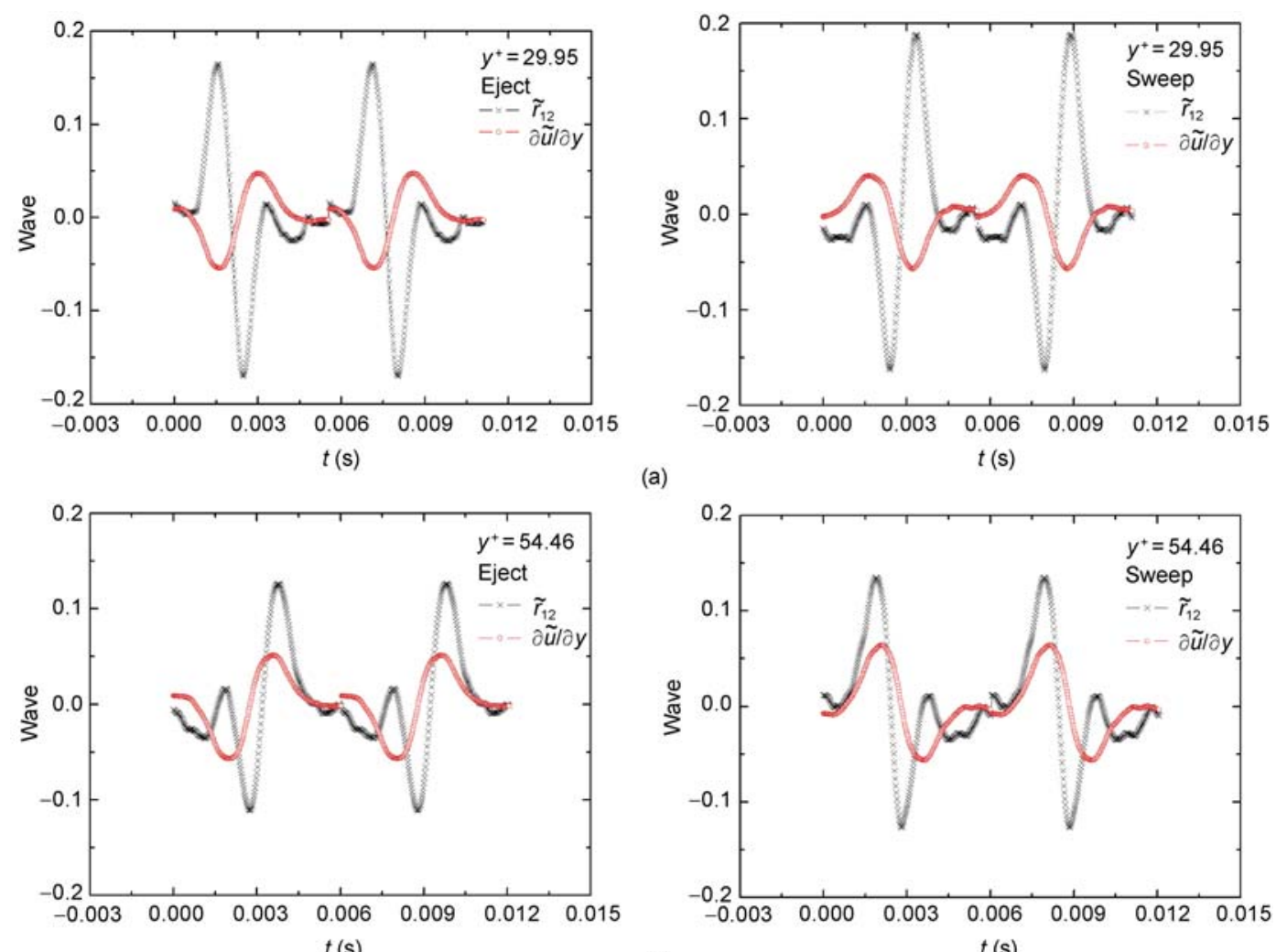

(a)
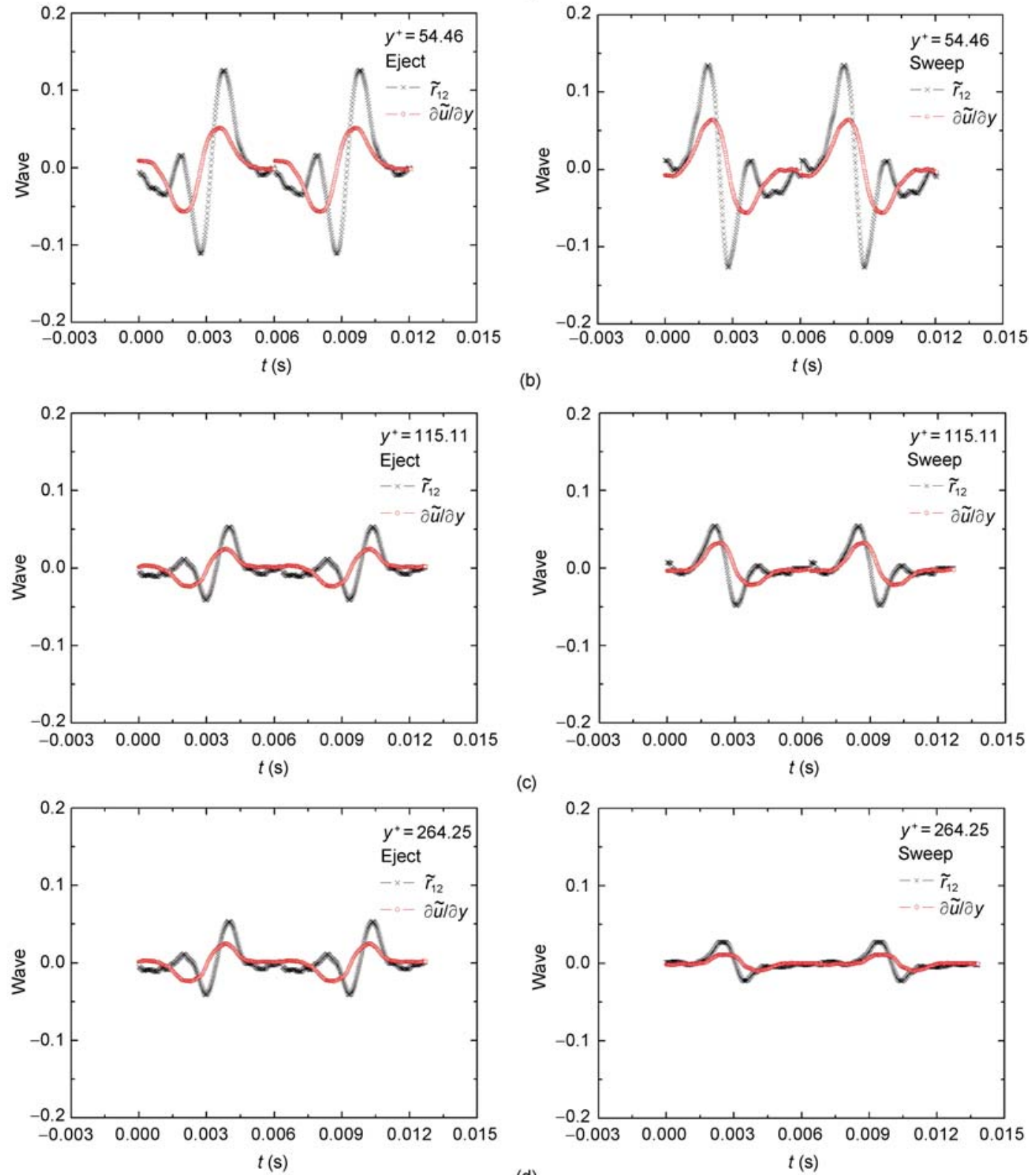

(c)

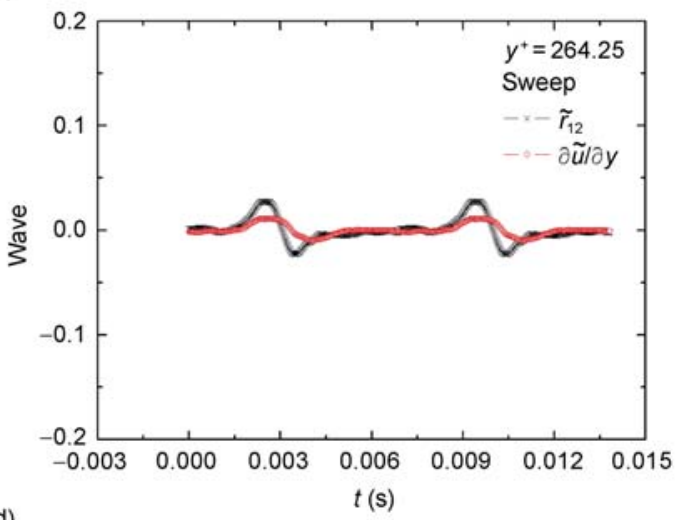

Figure 8 (Color online) Waveforms of $\tilde{r}_{12}$ and $\partial \tilde{u} / \partial y$ during the ejection and sweep process at several normal-wall locations for 8-th scale CS ( $x=800$ $\mathrm{mm})$. 
vect faster than $\partial \tilde{u} / \partial y$ of CS in spatial phase with the relative positive streamwise fluctuation velocity component $u^{\prime}>0$ and negative normal-wall fluctuation velocity component $v^{\prime}<0$. So in the spatial streamwise direction $\tilde{r}_{12}$ is located downstream of $\partial \tilde{u} / \partial y$. Equivalently in the temporal direction, the phase of $\tilde{r}_{12}$ is ahead of that of $\partial \tilde{u} / \partial y$ and $\tilde{r}_{12}$ can be captured by the hot-wire probe earlier than $\partial \tilde{u} / \partial y$ with $\varphi(y)>\theta(y)$ and $\Delta \Phi=\varphi(y)-\theta(y)>0$. Consequently, the relaxation effect of stress-strain should be related to the detailed physical process of CS burst. This effect should be taken into consideration for CEVM.

It can be seen from Figure 8(a)-8(c) that the phase difference between $\tilde{r}_{12}$ and $\partial \tilde{u} / \partial y$ gradually becomes small as the normal-wall location increases from $y^{+}=29.95$ to $y^{+}=115.11$. Figure $8(\mathrm{~d})$ shows the phase difference between them becomes very small at $y^{+}=264.25$. It means that the relaxation effect of $\tilde{r}_{12}$ and $\partial \tilde{u} / \partial y$ can be negligible out of TBL.

For a quantitative estimation of the time-phase difference between $\tilde{r}_{12}$ and $\partial \tilde{u} / \partial y$, correlation function between $\tilde{r}_{12}$ and $\partial \tilde{u} / \partial y$ is calculated as a function of time delay $\tau$. The correlation-function between $\tilde{r}_{12}$ and $\partial \tilde{u} / \partial y$ is defined as:

$$
R_{\tilde{r}_{1}, \partial \tilde{u} / \partial y}\left(a_{j}, \tau\right)=\int_{0}^{T}\left[\tilde{r}_{12}\left(a_{j}, t\right) \frac{\partial \tilde{u}\left(a_{j}, t+\tau\right)}{\partial y}\right] \mathrm{d} t,
$$

where $T$ refers to the period of 8 -th scale CS. The phase difference can be determined by the time delay $\tilde{\tau}$ corresponding to the maximum of $R_{\tilde{r}_{1}, \partial \tilde{u} / \partial y}$ :

$$
\begin{gathered}
R_{\tilde{r}_{1}, \partial \tilde{u} / \partial y}\left(a_{j}, \tilde{\tau}\right)=\max R_{\tilde{r}_{1}, \partial \tilde{u} / \partial y}\left(a_{j}, \tau\right), \\
\Delta \Phi_{j}=2 \pi \frac{\tilde{\tau}}{T_{j}} .
\end{gathered}
$$

To further ascertain the variation of phase difference between $\tilde{r}_{12}$ and $\partial \tilde{u} / \partial y$ across the TBL, we plot, in Figure 9 , the $\Delta \Phi$ as a function of $y^{+}$at two streamwise locations during the ejection and sweep process for 8-th scale CS. $|\Delta \Phi|$ shows a monotone decreasing function with a maximal value of $|\Delta \Phi|=70^{\circ}$ at $y^{+}=29.95$ and sharply decays to $20^{\circ}$ at $y^{+}=60.15$. If we extend the variation of the phase difference to the near-wall region, it can be prognosticated that the phase difference will be $|\Delta \Phi|=90^{\circ}$ at the wall, which means the eddy viscosity is a pure imaginary number. As $y+$ continues to increase faraway from the wall, $|\Delta \Phi|$ maintains constant about $20^{\circ}$ in the logarithm region. For $y^{+}$out of TBL, the phase difference $\Delta \Phi$ maintains $-20^{\circ}$ for the ejection case, while $\Delta \Phi$ gently decreases to $0^{\circ}$ for the sweep case. The reason is that CS hairpin vortex head is always inclined downstream at an angle of $12^{\circ}-13^{\circ}$ from the wall and the backward eject fluids always prevent small-scale fluctuation, as well as $\tilde{r}_{12}$, from coming close to the CS itself. So they always keep a fixed distance in the streamwise direction during ejection even in the outer region. So $\Delta \Phi$ maintains a small constant, $-20^{\circ}$, for the ejection case. But for the sweep case, the situation is quite different. The clockwise rotating fluids at the hairpin vortex head of CS can induce the small-scale fluctuation, as well as $\tilde{r}_{12}$, being attracted very close to the CS itself. This leads $\Delta \Phi$ to decrease to $0^{\circ}$.

Figure 9 demonstrates the phase difference information is relatively important in the near wall region. It provides the direct confirmation of $\Delta \Phi$ (namely, the relaxation effect of stress-strain) related to the detailed physical process of CS burst in the TBL.

\section{Conclusions}

Based on the theory of CEVM for CS, the waveforms of Reynolds stress residual contribution term of random fluctuations to CS and the velocity strain rate of CS are investigated experimentally by the conditional phase average technique and the cross-correlation function method. Furthermore, it is verified that the phase difference between $\tilde{r}_{12}$ and $\partial \tilde{u} / \partial y$ should be taken into account in the eddy viscosity model. The conclusions are as follows:

(1) Reynolds stress residual contribution term is not synchronized with the velocity strain rate of CS; there exists a phase difference between them both in temporal and spatial

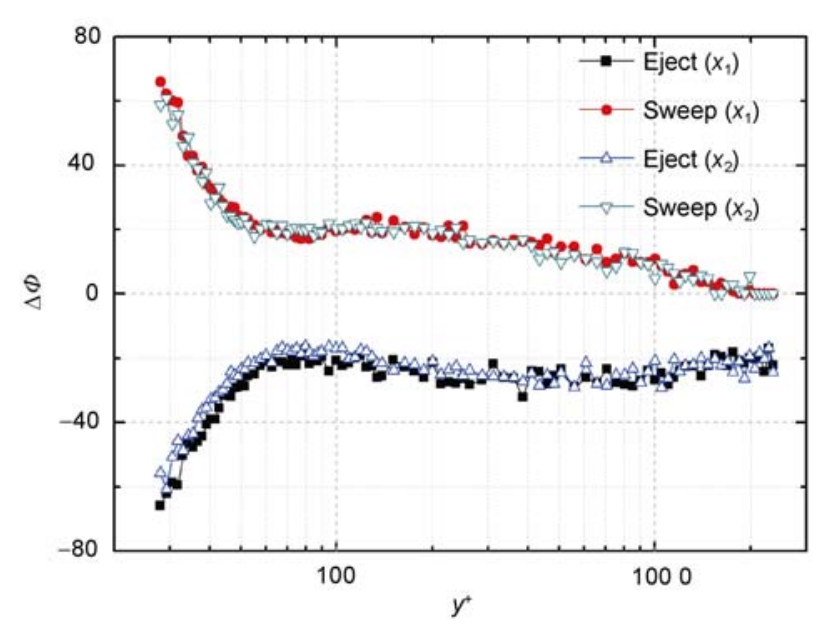

Figure 9 (Color online) Evolution of the phase differences between $\tilde{r}_{12}$ and $\partial \tilde{u} / \partial y$ across the TBL during the ejection and sweep process for 8-th scale CS at two streamwise locations $x_{1}=800 \mathrm{~mm}$ (solid symbol) and $x_{2}=820 \mathrm{~mm}$ (open symbol). 
space. The reason is the macro relaxation spatio-temporal scale of the momentum exchange between eddy structures should be no longer negligible in turbulent fields. Therefore, the hypothesis of CEVM for CS dynamics should be reasonable. These factors should also be taken into consideration in the turbulence model theory for unsteady and non-equilibrium turbulent flows.

(2) It has been experimentally verified that the phase difference between them is related to the detailed physical process of CS burst, such as ejection and sweep event. Reynolds stress residual contribution term lags temporally behind the velocity strain rate of CS during an ejection event, reversely during a sweep event.

(3) Furthermore, the near wall region is of the most importance for turbulence production in the TBL since the more actively CS burst in this region and the more remarkable relaxation effect than that in any other regions with the phase difference is $90^{\circ}$, which means the eddy viscosity is a pure imaginary number.

This work was jointly supported by the National Natural Science Foundation of China (Grant Nos. 10832001 and 10872145), and Opening Subject of State Key Laboratory of Nonlinear Mechanics, Institute of Mechanics, Chinese Academy of Sciences.

1 Reynolds W C, Hussain A K M F. The mechanics of an organized wave in turbulent shear flow. Part 3. J Fluid Mech, 1972, 54: 263-288

2 Wu X S, Zhou H. Linear instability of turbulent boundary layer as a mechanism for the generation of large scale coherent structures. Chin Sci Bull, 1989, 34: 1685-1690

3 Brown G L, Thomas A S W. Large structure in a turbulent boundary layer. Phys Fluids, 1977, 20: S243-S252

4 Falco R E. Coherent motions in the outer region of turbulent boundary layers. Phys Fluids, 1977, 20: S124-S132

5 Luo J S, Zhou H. The comparison of theoretical model with experiment on large scale coherent structures in outer field of turbulent boundary layer (in Chinese). Sci China Ser A-Math Phys Astron Tech, 1993, 36: 56-62

6 Antonia R A, Bisset D K, Browne L W B. Effect of Reynolds number on the topology of the organized motion in a turbulent boundary layer. J Fluid Mech, 1990, 213: 267-286

7 Hadzic I, Hanjalic K, Laurence D. Modeling the response of turbulence subjected to cyclic irrotational strain. Phys Fluids, 2001, 13: 1740-1747

8 Luo J S, Wang X J. The study of turbulent properties in stable flexuous blow-suck boundary channel flow (in Chinese). Chin J Theor Appl Mech, 2005, 37: 673-681

9 Bruns J M, Fernholz H H, Monkewitz P A. An experimental investigation of a three-dimensional turbulent boundary layer in an ' $\mathrm{S}$ ' shaped duct. J Fluid Mech, 1999, 393: 175-213

10 Wang X J, Luo J S, Zhou H. On the eddy viscosity model of periodic turbulent shear flows. Acta Mech Sin, 2003, 19: 470-475

11 Li W P, Wang X Q, Xie H. Phase delay of the Reynolds stress and wall pressure fluctuation in wall shear flows (in Chinese). J Huazhong Univ Sci Tech (Nat Sci), 2007, 35: 122-125

12 Guo A D, Jiang N. Experimental research on complex eddy viscosity modeling of multi-scale coherent structures in wall turbulence (in Chinese). Chin J Theor Appl Mech, 2010, 42: 159-168

13 Jiang N, Liu W, Liu J H, et al. Phase-averaged waveforms of Reynolds stress in wall turbulence during the burst events of coherent structures. Sci China Ser G-Phys Mech Astron, 2008, 51: 857-866

14 Farge M. Wavelet transforms and their applications to turbulence. Annu Rev Fluid Mech, 1992, 24: 395-457

15 Jiang N, Wang Z D, Shu W. The maximum energy criterion for identifying burst events in wall turbulence using wavelet analysis (in Chinese). Chin J Theor Appl Mech, 1997, 29: 406-412

16 Jiang N, Zhang J. Detecting multi-scale coherent eddy structures and intermittency in turbulent boundary layer by wavelet analysis. Chin Phys Lett, 2005, 22: 1968-1971 